\title{
Newly developed flexible endoscopic scissors for removal of a nasogastric tube trapped within a gastrojejunal anastomotic staple line
}

A 72-year-old woman underwent a laparorobotic pancreaticoduodenectomy using the da Vinci Surgical System (Intuitive Surgical Inc., Sunnyvale, California, USA) for removal of a 4-cm ampullary adenoma. Intraoperatively, a nasogastric tube was inserted into the gastric fundus and its proximal end was secured to the patient's nares. On postoperative day 2 , the tube could not be removed at the bedside.

Esophagogastroduodenoscopy revealed that the nasogastric tube was trapped along the gastrojejunostomy anastomotic staple line by a few staples ( $\mathbf{F i g} \mathbf{1}$ ).

Initial attempts to completely liberate this portion of the tube were unsuccessful despite the use of multiple mechanical modalities (such as rat-tooth forceps, loop wire cutters, snares, and standard endoscopic scissors) in combination with monopolar and bipolar electrocautery (॰ Fig. 2).

Finally, a pair of flexible endoscopic scissors, FlexShears (Apollo Endosurgery Inc., Austin, Texas, USA) $($ Fig. 3), were inserted into the side hole of the nasogastric tube and used to completely cut though the tube as it was dislodged from the staple line ( $\bullet$ Fig. 4; $\bullet$ Video 1 ).

No evidence of subsequent disruption or dehiscence at the anastomosis was observed, and the patient continued her postoperative recovery without further complications or the need for further operations.

Comparable scissors-type devices have been used to aid in endoscopic submucosal dissection of both gastric [1] and colorectal [2] neoplasms, but these devices function more as grasping forceps than actual cutting shears. Karmali et al. reported the successful use of the Olympus straight endoscopic scissors device to remove eroded laparoscopically placed gastro-

\section{Video 1}

Traction was applied to the nasogastric tube using forceps. Argon plasma coagulation (APC) was then used in an effort to degrade or dislodge the tube. Finally, the FlexShears flexible endoscopic scissors were used to free the tube from the staple line.

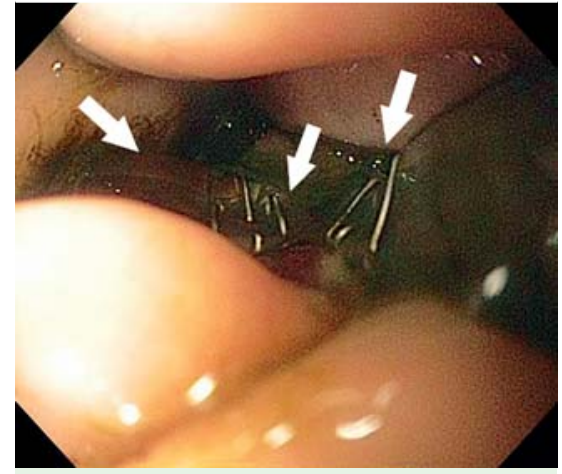

Fig. 1 Distal end of the nasogastric tube (arrows) stapled into the gastrojejunostomy anastomosis.

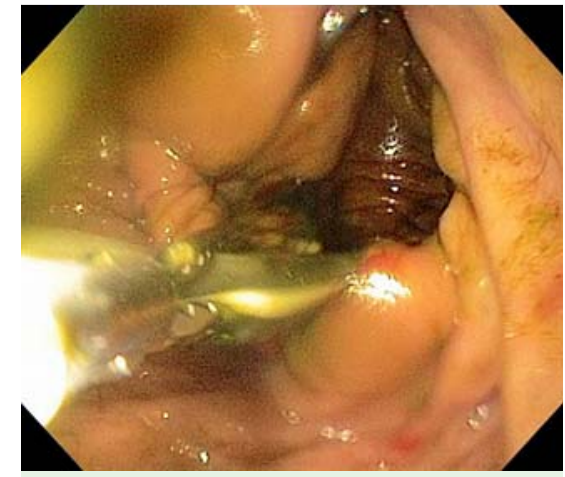

Fig. 2 Rat-tooth forceps during an unsuccessful attempt to dislodge the nasogastric tube.

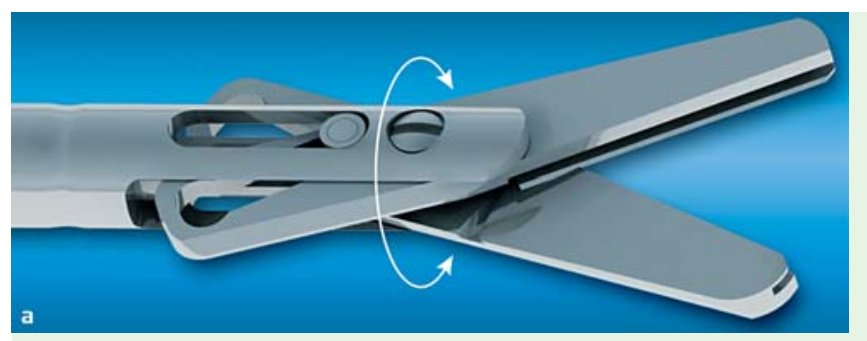

Fig. 3 a, b FlexShears flexible endoscopic scissors. (Reproduced with the permission of Apollo Endosurgery, Inc.)

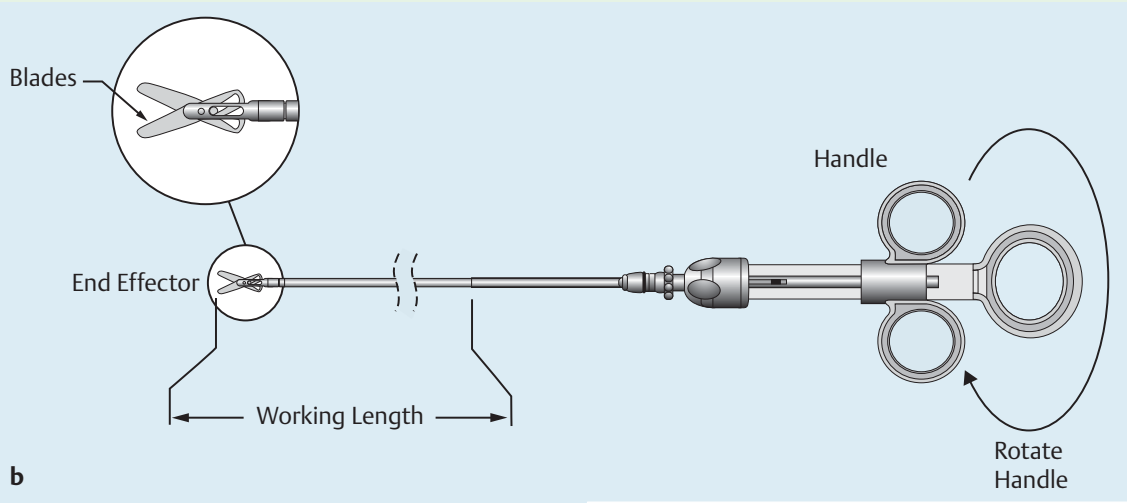

plasty bands in nine patients with obstructive symptoms [3]. The FlexShears described in the current report act as single-use flexible cutting shears designed for tissue dissection during flexible endoscopic procedures. This is the first reported case in humans demonstrating safe and effective use of these flexible endoscopic scissors since their approval by the Food and Drug Administration (FDA) in 2009.

Endoscopy_UCTN_Code_TTT_1AO_2AD

Competing interests: None

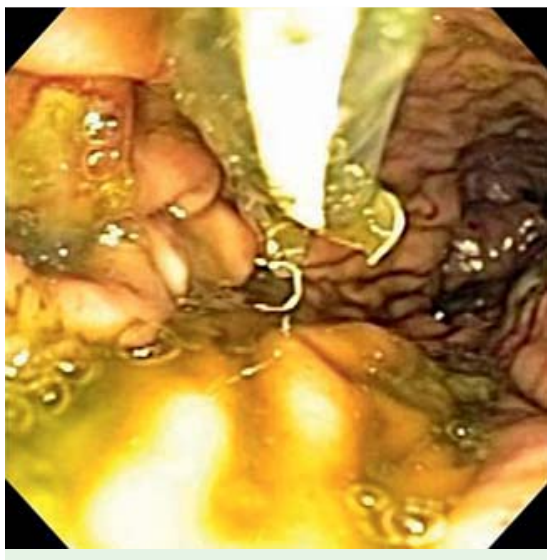

Fig. 4 The cut end of the nasogastric tube as it was pulled free from the staple line. 
M. N. Wilkinson ${ }^{1}$, V. Jayaraman ${ }^{1}$, K. Watkins ${ }^{2}$, P. Bao ${ }^{2}$, J. M. Buscaglia ${ }^{1}$ 1 Department of Medicine Stony Brook University Medical Center, State University of New York at Stony Brook, Stony Brook, New York, USA

2 Department of Surgery, Stony Brook University Medical Center, State University of New York at Stony Brook, Stony Brook, New York, USA

\section{References}

1 Akahoshi K, Honda K, Akahane H et al. Endoscopic submucosal dissection by using a grasping-type scissor forceps: a preliminary clinical study. Gastrointest Endosc 2008; 67: $1128-1133$

2 Honma K, Kobayash M, Watanabe Het al. Endoscopic submucosal dissection for colorectal neoplasia. Dig Endosc 2010; 22: $307-$ 311

3 Karmali S, Snyder B, Wilson EB et al. Endoscopic management of eroded prosthesis in vertical banded gastroplasty patients. Surg Endosc 2010; 24: 98 - 102
Bibliography

DOI $10.1055 / \mathrm{s}-0030-1256426$

Endoscopy 2011; 43: E234-E235

(c) Georg Thieme Verlag KG Stuttgart · New York . ISSN 0013-726X

\section{Corresponding author}

\section{Wilkinson, MD}

Division of Gastroenterology

Stony Brook University Medical Center

100 Nicolls Road

HSC Level 17, Room 060

Stony Brook

New York 11794

USA

Fax: +1-631-444-8886

mwilkinson@notes.cc.sunysb.edu 